\title{
Exact, almost and delayed fault detection an observer based approach
}

\section{Niemann, Hans Henrik; Saberi, Ali; Stoorvogel, Anton A.; Sannuti, Peddapullaiah}

\section{Published in:}

Proceeding of the American Control Conference

Link to article, DOI:

10.1109/ACC.1999.782748

Publication date:

1999

Document Version

Publisher's PDF, also known as Version of record

Link back to DTU Orbit

Citation (APA):

Niemann, H. H., Saberi, A., Stoorvogel, A. A., \& Sannuti, P. (1999). Exact, almost and delayed fault detection: an observer based approach. In Proceeding of the American Control Conference (Vol. 1, pp. 99-103) https://doi.org/10.1109/ACC.1999.782748

\section{General rights}

Copyright and moral rights for the publications made accessible in the public portal are retained by the authors and/or other copyright owners and it is a condition of accessing publications that users recognise and abide by the legal requirements associated with these rights.

- Users may download and print one copy of any publication from the public portal for the purpose of private study or research.

- You may not further distribute the material or use it for any profit-making activity or commercial gain

- You may freely distribute the URL identifying the publication in the public portal 
Exact, Almost and Delayed Fault Detection An Observer based Approach

\author{
Henrik Niemann ${ }^{1}$ \\ Department of Automation \\ Technical University of Denmark \\ Building 326, DK-2800 Lyngby, Denmark \\ E-mail: hhn@iau.dtu.dk \\ Anton A. Stoorvogel \\ Dept of Math. and Computing Science \\ Eindhoven Univ. of Technology \\ P.O.Box 513, $5600 \mathrm{MB}$ Eindhoven \\ The Netherlands \\ E-mail: wscoas@win.tue.nl
}

\begin{abstract}
This paper considers the problem of fault detection and isolation while using zero or almost zero threshold. A number of different fault detection and isolation problems using exact or almost exact disturbance decoupling are formulated. Solvability conditions are given for the formulated design problems. The $\ell$-step delayed fault detection problem is also considered for discrete-time systems.
\end{abstract}

\section{Introduction}

This paper is concerned with fault detection and isolation (FDI) in dynamic systems. The increasing use of fault detectors in different connections has stimulated over the last two decades an extensive study of fault detection and isolation methods. Some of the first fault detection methods have been described in a survey paper by Willsky [10]. A recent paper by Patton and Chen [5] give a good overview of more modern model-based fault detection methods. Such a study has been enhanced by the developments in modern control theory that has brought forth powerful techniques of mathematical modeling, $H_{2}$ and $H_{\infty}$ optimal control, state estimation, parameter identification, etc.

Fault detection can be done in a number of different levels, where the high level fault detection is an exact estimation of the faults and the low level one is just an indication of faults somewhere in the system [10]. High level fault detection is in general achieved by applying observer based fault detectors.

In a model based approach, most fault detectors have been based on observers, see e.g. [4]. By using an observer as a fault detector, the fault detection design prob-

\footnotetext{
${ }^{1}$ Supported by the Danish Technical Research Council under grant no. 96-01557.
}

\author{
Ali Saberi \\ School of Electrical Engineering \\ Washington State University \\ Pullman, WA 99164-2752, USA \\ E-mail: saberi@eecs.wsu.edu \\ Peddapullaiah Sannuti \\ Dept. of Elec. and Computer Eng. \\ Rutgers University \\ 94 Brett Road, Piscataway \\ NJ 08854-8058, USA \\ E-mail: sannuti@ece.rutgers.edu
}

lem more or less reduce to an estimation problem.

In the paper of Massoumnia et.al. [2], a number of fundamental problems in fault detection are considered. These fundamental problems are characterized by using the direction of the fault vector. These problems are important, because if they can be solved, it is possible to apply fault detectors with zero or almost zero threshold. As is well known, the selection of thresholds is in general a major problem as it always involves some trade-offs between false alarms and non-detected faults as soon as the threshold is unequal to zero $[1,6]$.

The focus of this paper is to examine the fundamental problems of fault detection and isolation based on a direct estimation of the fault vector which is obviously a subset of the fundamental problems formulated in [2]. Fault detection and isolation problems without using a direct estimation of the fault vector are considered in [8]. We give the solvability conditions of the considered problems in a general setup.

Both continuous as well as discrete-time systems are considered in this paper. We would like to emphasize that the design problems that are considered in this paper are very general and are very interesting from a theoretical point of view.

A full version of this paper with proofs can be found in [3].

\section{Definitions and Problem Formulations}

Consider the following state space description for a plant or a system given by

$$
\Sigma:\left\{\begin{aligned}
\sigma x & =A x+B_{f} f+B_{\mu} \mu \\
y & =C x+D_{f} f+D_{\mu} \mu
\end{aligned}\right.
$$

where $\sigma$ is an operator indicating the time derivation $\frac{d}{d t}$ for continuous-time systems and a forward unit time shift for discrete-time systems. Also, $x$ is the state vec- 
tor, $f$ is a fault signal vector, $\mu$ is a disturbance signal vector, and $y$ is the measurement vector.

The above can be rewritten in a transfer function form as

$$
y(\alpha)=G_{f}(\alpha) f(\alpha)+G_{\mu}(\alpha) \mu(\alpha)
$$

where $\alpha$ is the Laplace operator $s$ for continuous-time systems and the $z$-transform $z$ for discrete-time systems.

Various types of fault detection and isolation problems are defined rigorously later on in this section. In all such problems, fault isolation consists of essentially generating what is known as a residual signal or a residual vector $r$ which is then utilized to detect and isolate a fault. The residual $r$ is generated from the measured output $y$ of the given process by a dynamic system known as a residual generator whose transfer function is $F$,

$$
r(\alpha)=F(\alpha) y(\alpha)
$$

In what follows, we first formally define what we mean by fault detection and isolation, and then pose four types of fault isolation problems. Solvability conditions for such problems and methods of designing appropriate residual generators are considered in the subsequent sections. Before we proceed to the definitions, we introduce the notation,

$$
r=F G_{f} f+F G_{\mu} \mu=T_{r f} f+T_{r \mu} \mu .
$$

We now start with the definition of fault detection and isolation.

Definition 1 Given the residual generator $F \in \mathcal{R} \mathscr{H}_{\infty}$, the residual $r$ is said to achieve fault detection (FD) without disturbance if a non-zero fault vector $f$ and $\mu=0$ results in a non-zero residual $r$.

Definition 2 Given the residual generator $F \in \mathcal{R} \mathscr{H}_{\infty}$, the residual $r$ is said to achieve fault detection and isolation $(F D I)$ without disturbance if for any two different fault vectors $f_{i}$ and $f_{j}$ and $\mu=0$ the corresponding residuals $r_{i}$ and $r_{j}$ are different.

Definitions 1 and 2 are elementary and basic definitions concerned with fault detection and isolation. The real issue is however whether the above can still be achieved when the disturbance $\mu$ affects the system. In other words, we need to have a residual generator which is insensitive to external disturbance $\mu$. That is, some specified norm of $T_{r \mu}$ has to be small.

As argued by Willsky (see [10]) a very desirable case is if we can reconstruct the fault signal $f$ using a suitable observer. Willsky considered the case that this reconstruction should be exact even in the presence of the disturbances. In this paper we will consider two relaxations which will turn out to weaken significantly the solvability conditions. First of all we allow for almost reconstruction but with an arbitrarily high level of accuracy. Secondly we consider the case that the reconstruction can be performed using delayed measurements.

There are many definitions of fault detection and isolation (see e.g. $[2,5,9]$ ) and corresponding problems in the literature. The one posed here turns out (see for details the next section) to be quite fundamental. A more general and structural analysis of the problems in fault detection and analysis will be considered in another paper, see [8].

In what follows, we pose two different problems each imposing certain specified and desired conditions on $Q T_{r \mu}$ and $Q T_{r f}$, where $Q$ is a diagonal matrix with 0 or 1 on the diagonal which describes the subset of faults that are going to be detected and isolated. Note that $Q=I$ is a special case when all fault are detected and isolated. The discrete-time versions of the following design problems appear by using $e^{j \omega}$ instead of $j \omega$.

Problem 1 The problem of partial exact fault detection and isolation (PEFDI Problem) is defined as a problem of finding, if existent, a residual generator $F \in \mathcal{R}_{\infty}$ such that we have

$$
Q T_{r f}(j \omega)=Q I \text { and } Q T_{r \mu}(j \omega)=0 \forall \omega \in \mathbb{R} .
$$

Clearly, the exact fault detection and isolation problem imposes strong conditions. If it is solvable, any fault can be detected without any false alarm. In fact, the residual signal then will be a perfect estimate of the fault. As such, exact fault detection and isolation may not always be solvable. The following problem addresses almost exact fault detection and isolation which seeks to relax the perfect estimation condition imposed in the exact fault detection and isolation problem.

Problem 2 The problem of partial almost exact fault detection and isolation (PAFDI Problem) is defined as a problem of finding, if existent, a parameterized family of residual generators $F_{\rho} \in \mathcal{R} \mathcal{H}_{\infty}$ parameterized in $\rho$ such that for any $\varepsilon>0$ there exists a $\rho^{*}$ such that for any $\rho<\rho^{*}$ we have

$$
\left\|Q\left(T_{r f, \rho}-I\right)\right\|_{H}<\varepsilon \text { and }\left\|Q T_{r \mu, \rho}\right\|_{H}<\varepsilon,
$$

where, as usual, $\|\cdot\|_{H}$ is a suitable norm. Here, $T_{r f . \rho}$ and $T_{r \mu, \rho}$ correspond to the expressions given in (3) for $T_{r f}$ and $T_{r \mu}$ respectively with $F_{\rho}$ replacing $F$.

In connection with Problem 2, one can use any norm. Norms that are commonly used in the literature are the $H_{2}$ and $H_{\infty}$ norms. But one can also use the so-called $L_{1}$ norm that measures the $L_{\infty}$ induced operator norm.

A time delay in detecting faults in a dynamic system can be accepted in a large number of cases. This will, of 
course, depend on the type of fault to be detected and in which connection the information is going to be used.

For discrete-time systems, let us consider the $\ell$-step delayed fault detection problem. In an $\ell$-step fault detector, we get a detection of the fault signal $f(k-\ell)$ rather than $f(k)$ at time $k$. The exact and almost exact fault detection design problems given above can now be rewritten for the case when a $\ell$-step delayed fault detector is applied. The problem formulation given above was based on making the estimation error $e_{Q}(k)$ given by

$$
e_{Q}(k)=Q(f(k)-r(k))
$$

equal to zero or almost equal to zero independent of the inputs as time goes to infinity. In the $\ell$-step delayed fault detection case, the equation for the estimation error in (4) takes then the form,

$$
e_{Q . \ell}(k)=Q(f(k-\ell)-r(k)) .
$$

Based on this equation, we get the following two $\ell$-step delayed fault detection problems.

Problem 3 The problem of partial exact fault detection and isolation with $\ell$-step delay $(\ell$-step delayed PEFDI Problem) is defined as a problem of finding, if existent, a residual generator $F \in \mathcal{R H}_{\infty}$ such that we have

$$
\begin{aligned}
& Q T_{r f}\left(e^{j \omega}\right)=e^{-j \ell \omega} Q I \text { and } \\
& Q T_{r \mu}\left(e^{j \omega}\right)=0 \quad \forall \omega \in[0,2 \pi) .
\end{aligned}
$$

Problem 4 The problem of partial almost exact fault detection and isolation with $\ell$-step delay ( $\ell$-step delayed PAFDI Problem) is defined as a problem of finding, if existent, a parameterized family of residual generators $F_{\rho} \in \mathcal{R} \mathscr{H}_{\infty}$ parameterized in $\rho$ such that for any $\varepsilon>0$ there exists a $\rho^{*}$ such that for any $\rho<\rho^{*}$ we have

$$
\left\|Q\left(T_{r f, \rho}-J\right)\right\|_{H}<\varepsilon \text { and }\left\|Q T_{r \mu, \rho}\right\|_{H}<\varepsilon,
$$

where $J(j \omega)=e^{-i \ell \omega} I$ and, as usual, $\|\cdot\|_{H}$ is a suitable norm. Here, $T_{r f, \rho}$ and $T_{r \mu, \rho}$ correspond to the expressions given in (3) for $T_{r f}$ and $T_{r \mu}$ respectively, with $F_{\rho}$ replacing $F$.

\section{Analysis of and Design for Solving (Partial) Exact or Almost Exact Fault Detection and Isolation}

In this section, we will develop the necessary and sufficient conditions under which (partial) exact or almost exact fault detection and isolation is possible.

Since all four problems are related to estimation theory, it is natural to come up with a mathematically unified framework to develop the solvability conditions for them as well as to construct appropriate residual generators solving them.
Before we proceed further we need some notation. Let

$$
B:=\left(\begin{array}{ll}
B_{f} & B_{\mu}
\end{array}\right), \quad D:=\left(\begin{array}{ll}
D_{f} & D_{\mu}
\end{array}\right) .
$$

Also, denote the output variable that is to be estimated as

$$
z:=Q f=\bar{Q}\left(\begin{array}{l}
f \\
\mu
\end{array}\right) \text { where } \bar{Q}:=\left(\begin{array}{ll}
Q & 0
\end{array}\right) .
$$

Further, let us define

$$
\tilde{C}=\left(\begin{array}{ll}
C & D
\end{array}\right), \tilde{Q}=\left(\begin{array}{ll}
0 & \bar{Q}
\end{array}\right)
$$

Without the loss of generality, we use the following assumption throughout this section.

Assumption 3 The eigenvalues of $A$ are in the open lefthalf complex plane for continuous-time systems, and are inside the unit circle for discrete-time systems.

The argument why the above definition is without loss of generality can for instance be found in [7].

\subsection{Solvability Conditions for (Partial) Exact or Al- most Exact Fault Detection and Isolation Problems}

In this subsection, we present the solvability conditions for the (partial) exact or almost exact fault Detection and isolation problems.

An observer in general has the form,

$$
\begin{aligned}
\sigma v & =L v+M y \\
\hat{z} & =N v+P y .
\end{aligned}
$$

From [7], we have that the partial exact or almost exact fault detection and isolation problem can only be solved by utilizing only a proper (as opposed to a strictly proper) observer, and even then one must at least satisfy the necessary condition:

$$
\operatorname{Ker} D \subseteq \operatorname{Ker} \bar{Q} \text {. }
$$

The following two theorems establish the necessary and sufficient conditions under which the partial exact or almost exact fault detection and isolation problems for continuous- and discrete-time systems can be solved. For the definitions of the geometric subspaces $\jmath^{*}, \mathcal{V}^{*}$, etc., we refer to any standard textbook on geometric theory of linear systems.

Theorem 4 Consider the continuous-time system $\Sigma$ given in (I), and characterized by the quadruple $(A, B, C, D)$ with the eigenvalues of $A$ in the open lefthalf plane. We have the following:

1. The partial exact fault detection and isolation problem is solvable if and only if

$$
\left(s^{-}(\Sigma) \oplus \mathbb{R}^{(m+s)}\right) \cap \operatorname{Ker} \tilde{C} \subseteq \operatorname{Ker} \tilde{Q}
$$


2. The partial almost exact fault detection and isolation problem while utilizing the $\mathrm{H}_{2}$ norm is solvable if and only if

$$
\begin{array}{r}
\left(\left[s^{-()}(\Sigma) \cap \mathcal{v}^{*}(\Sigma)\right] \oplus \mathbb{R}^{(m+s)}\right) \cap \operatorname{Ker} \tilde{C} \\
\subseteq \operatorname{Ker} \tilde{Q} .
\end{array}
$$

3. The partial almost exact fault detection and isolation problem while utilizing the $H_{\infty}$ norm is solvable if and only if the following conditions are satisfied.

(a) (9) is satisfied,

(b) for any invariant zero $s_{0}$ of $\Sigma$ on the imaginary axis we have

$$
\operatorname{Ker} C\left(s_{0} I-A\right)^{-1} B+D \subset \operatorname{Ker} \bar{Q} .
$$

We emphasize that the above theorem can easily be used to find the solvability conditions for the exact fault detection and isolation problems by defining $\bar{Q}$ as $\left(\begin{array}{ll}I_{m} & 0\end{array}\right)$. Next, consider the discrete-time version of the above theorem.

Theorem 5 Consider the discrete-time system $\Sigma$ given in (I), and characterized by the quadruple $(A, B, C, D)$ with the eigenvalues of $A$ inside the unit disc. We have the following:

1. The partial exact fault detection and isolation problem is solvable if and only if

$$
\left(s^{\ominus}(\Sigma) \oplus \mathbb{R}^{(m+s)}\right) \cap \operatorname{Ker} \tilde{C} \subseteq \operatorname{Ker} \tilde{Q} .
$$

2. The partial exact fault detection and isolation problem while utilizing the $\mathrm{H}_{2}$ norm is solvable if and only if

$$
\left(s^{\otimes}(\Sigma) \oplus \mathbb{R}^{(m+s)}\right) \cap \operatorname{Ker} \tilde{C} \subseteq \operatorname{Ker} \tilde{Q}
$$

3. The partial exact fault detection and isolation problem while utilizing the $H_{\infty}$ norm is solvable if and only if the following conditions are satisfied.

(a) (10) is satisfied,

(b) For any invariant zero $s_{0}$ of $\Sigma$ on the unit circle we have

$$
\operatorname{Ker} C\left(s_{0} I-A\right)^{-1} B+D \subset \operatorname{Ker} \bar{Q} .
$$

3.2 Solvability Conditions for $\ell$-step Delayed (Partial) Exact or Almost Exact Fault Detection and Isolation problems

In order to give the necessary and sufficient solvability conditions for the $\ell$-step delayed fault detection problems, the estimation error given in (5) needs to be in a state space form. Denoting the output variable that is to be estimated by $z$, the $\ell$-step delayed fault detection problem for the original system is equivalent with the design of a proper fault detector for the following system

$$
\begin{aligned}
\bar{x}(k+1) & =\bar{A} \bar{x}(k)+\bar{B}\left(\begin{array}{l}
f(k) \\
\mu(k)
\end{array}\right) \\
y(k) & =\bar{C}_{1} \bar{x}(k)+D\left(\begin{array}{l}
f(k) \\
\mu(k)
\end{array}\right) \\
z(k) & =\bar{C}_{2} \bar{x}(k),
\end{aligned}
$$

with

$$
\begin{aligned}
\bar{A} & =\left(\begin{array}{cccccc}
A & 0 & 0 & \cdots & 0 & 0 \\
0 & 0 & 0 & \cdots & 0 & 0 \\
I & 0 & 0 & \cdots & 0 & 0 \\
0 & I & 0 & \cdots & 0 & 0 \\
\vdots & \ddots & \ddots & \ddots & \vdots & \vdots \\
0 & \cdots & 0 & I & 0 & 0
\end{array}\right) \\
\bar{B}^{T} & =\left(\begin{array}{llllll}
B^{T} & \bar{Q}^{T} & 0 & \cdots & 0 & 0
\end{array}\right) \\
\bar{C}_{1} & =\left(\begin{array}{llllll}
C & 0 & 0 & \cdots & 0 & 0
\end{array}\right) \\
\bar{C}_{2} & =\left(\begin{array}{llllll}
0 & 0 & 0 & \cdots & 0 & I
\end{array}\right)
\end{aligned}
$$

where $\bar{x} \in \mathbb{R}^{(n+m+x) \ell}$ assuming that the original system has a state space of dimension $n$ and input space of order $m+s$ (for $f$ and $\mu$ ). Also, the definition of $B, D$ and $z$ are as given in (6) and (7).

The $\ell$-step delayed fault detection problem is now equivalent to designing a fault detector for the extended system given in (11) that will satisfy either exact or almost exact fault detection. However, these fault detection design problems are not exactly equivalent with the problems solved in Section 3. The reason is that the fault detection problem is now not an estimation of the external fault directly, but instead an estimation of a subset of the state vector described by $z$. As a consequence of this, it is possible also to apply strictly proper fault detectors for this setup. Furthermore, the solvability conditions given in Section 3 cannot be applied directly, because the design problem is now a state estimation problem instead of an input estimation problem.

It can be easily seen that using proper fault detectors when $\ell$-step delay is introduced in estimation is equivalent to using strictly proper fault detectors when $\ell+1$ step delay is introduced in estimation. Therefore we will only focus on using proper fault detectors in the following results. Based on the recent work on estimation theory [7], our study of $\ell$-step delayed partial exact and 
almost fault detection and isolation problems yields the following results.

Theorem 6 Consider the discrete-time system $\Sigma$ given in (I), and characterized by the quadruple $(A, B, C, D)$ with the eigenvalues of $A$ inside the unit disc. Let an integer $\ell \geq 0$ be given. We have the following:

1. The $\ell$-step delayed partial exact fault detection and isolation problem is solvable using a proper fault detector if and only if

$$
\begin{aligned}
& \left(s^{\ominus}(\Sigma) \oplus \mathbb{R}^{(m+s)}\right) \cap \operatorname{Ker}\left(\begin{array}{ll}
C & D
\end{array}\right) \\
& \cap\left(\begin{array}{ll}
A & B
\end{array}\right)^{-1} \mathcal{V}_{\ell}^{*}(\Sigma) \subseteq \operatorname{Ker}\left(\begin{array}{ll}
0 & \bar{Q}
\end{array}\right)
\end{aligned}
$$

2. The $\ell$-step delayed partial almost exact fault detection and isolation problem while utilizing the $\mathrm{H}_{2}$ norm is solvable using a proper fault detector if and only if

$$
\begin{gathered}
\left(s^{\otimes}(\Sigma) \oplus \mathbb{R}^{(m+s)}\right) \cap \operatorname{Ker}\left(\begin{array}{ll}
C & D
\end{array}\right) \\
\cap\left(\begin{array}{ll}
A & B
\end{array}\right)^{-1} \mathcal{V}_{\ell}^{*}(\Sigma) \subseteq \operatorname{Ker}\left(\begin{array}{ll}
0 & \bar{Q}
\end{array}\right)
\end{gathered}
$$

3. The $\ell$-step delayed partial almost exact fault detection and isolation problem while utilizing the $H_{\infty}$ norm is solvable using a proper fault detector if and only if the following conditions are satisfied.

(a) (12) is satisfied,

(b) For any invariant zero $s_{0}$ of $\Sigma$ on the unit circle we have

$$
\operatorname{Ker} C\left(s_{0} I-A\right)^{-1} B+D \subset \operatorname{Ker} \bar{Q} .
$$

Since we know that $\mathcal{V}_{\ell}^{*}(\Sigma)=\mathcal{V}^{*}(\Sigma)$ for $\ell \geq 0$, Theorem 6 can be rewritten by using $\mathcal{V}^{*}(\Sigma)$ instead of $\mathcal{V}_{\ell}^{*}(\Sigma)$.

\section{Conclusion}

For both continuous- and discrete-time systems, a number of fundamental problems that arise in fault detection and isolation (FDI) are investigated via estimation theory utilizing various types of observers or estimators.

Our primary focus is on solving exact and almost exact fault detection and isolation problems by direct estimation of fault signals. Exact estimation of a fault signal requires that the transfer function from the fault signal to its estimate is an identity matrix, while the transfer function from any disturbance to the estimate of the fault signal is identically zero. In contrast to this, almost estimation of a fault signal requires that the transfer function from the fault to its estimate be as close as desired to the identity matrix in a certain norm $\left(H_{2}\right.$ or $\left.H_{\infty}\right)$, while a chosen norm $\left(H_{2}\right.$ or $\left.H_{\infty}\right)$ of the transfer function from any disturbance to the estimate of the fault signal is as small as required. Necessary and sufficient conditions for the solvability of the exact fault detection and isolation problem as well as the almost exact fault detection and isolation problem are established. The notion of almost exact fault detection and isolation, as opposed to the exact fault detection and isolation, weakens the required solvability conditions considerably. Furthermore, for discrete-time systems, another significant notion of using a fixed delay in estimating a fault signal is introduced here. That is, at time step $k$, one obtains the estimate of the fault signal at $k-\ell$ where $\ell$ is a fixed nonnegative integer. Again, introduction of such a delay weakens the required solvability conditions.

\section{References}

[1] A. Emami-Naeini, M.M. Akhter, and S.M. Rock. Effect of model uncertainty on failure detection: The threshold selector. IEEE Transactions on Automatic Control, 33:1106-1115, 1988.

[2] M. Massoumnia, G.C. Verghese, and A.S. Willsky. Failure detection and identification. IEEE Transactions on Automatic Control, 34:316-321, 1989.

[3] H.H. Niemann, A. Saberi, A.A. Stoorvogel, and P. Sannuti. Exact, almost and delayed fault detection An observer based approach. International Journal of Robust and Nonlinear Control, 1999.

[4] R. Patton, P. Frank, and R. Clark. Fault diagnosis in dynamic systems - Theory and application. Prentice Hall, 1989.

[5] R.J. Patton and J. Chen. Robust fault detection and isolation (FDI) systems. Control and Dynamic Systems, 74:171-224, 1996.

[6] M.L. Rank and H.H. Niemann. Norm based design of fault detectors. International Journal of Control, 1999.

[7] A. Saberi, P. Sannuti, and A. Stoorvogel. Exact and almost input decoupled observers, inverse filtering, and deconvolution. Submitted for publication, 1999.

[8] A. Saberi, A. Stoorvogel, P. Sannuti, and H.H. Niemann. Fundamental problems in fault delection. Under preparation, 1998.

[9] J.E. White and J.L. Speyer. Detection filter design: Spectral theory and algorithms. IEEE Transactions on Automatic Control, 32:593-603, 1987.

[10] A.S. Willsky. A survey of design methods for failure detection in dynamic systems. Automatica, 12:601611,1976 . 